\title{
Modeling co-dynamics of pneumonia and meningitis diseases
}

\section{Getachew Teshome Tilahun ${ }^{1 *}$ (D)}

*Correspondence: gmgech@gmail.com

'Department of Mathematics, Haramaya University, Haramaya, Ethiopia

\section{Springer}

\begin{abstract}
In this paper an SIR deterministic mathematical model for co-infection of pneumonia and meningitis is proposed. We use a seven compartment model by using ordinary differential equations. The positivity of future solution of the model, the invariant region, and disease-free as well as endemic equilibrium are studied. To study the stability of the equilibria, a basic reproduction number is obtained by using the next generation matrix. The robustness of the model is also investigated. To identify the effect of each parameter on the expansion or control of the diseases, sensitivity analysis is performed. The effects of treating pneumonia infected only, meningitis infected only, and co-infected individuals have been identified by using the numerical simulation.
\end{abstract}

Keywords: Co-infection; Pneumonia; Meningitis; Stability; Numerical simulation

\section{Introduction}

Pneumonia, which can be categorized as one of airborne diseases, claims for the death of millions of human beings through inhaling pathogenic organism, mainly Streptococcus pneumoniae [1]. These bacteria are also responsible for the cause of other diseases such as meningitis, ear infections, and sinus infections. The diseases can affect all stages of human beings, from children to elderly people, and pneumonia becomes dangerous when the immunity level is lowered, and also in children or elderly people, as well as when it is co-infected with other diseases like meningitis [2]. Meningitis, which is an infection of the covering around the brain and spinal cord, can be both bacterial and viral. Bacterial infection of meningitis is the most common one; particularly, Streptococcus pneumoniae, haemophilus influenza, and Neisseria meningitidis are responsible for $80 \%$ of meningitis cases [3]. A lot of studies have been conducted to indicate controlling mechanisms of infectious diseases in a community, some of them are [4-6]. Mathematical modeling has a great role in describing the dynamics of infectious diseases in a community. Several scholars have developed different models to study the dynamics of different epidemic diseases. Some of them $[7,8]$ proposed a mathematical model of pneumonia and meningitis; moreover, [9] investigated the co-dynamics of pneumonia and typhoid fever diseases with optimal control and cost-effectiveness analysis. The result of the study revealed that prevention of typhoid fever and treatment of pneumonia is the most cost-effective strategies from the proposed ones. Different authors have studied co-infection of various diseases: [10] studied co-infection of HIV and pneumonia, [11] studied co-infection of pneumonia

(c) The Author(s) 2019. This article is distributed under the terms of the Creative Commons Attribution 4.0 International License (http://creativecommons.org/licenses/by/4.0/), which permits unrestricted use, distribution, and reproduction in any medium, provided you give appropriate credit to the original author(s) and the source, provide a link to the Creative Commons license, and indicate if changes were made. 
and malaria. However, to the best of our knowledge, no one has investigated co-infection of pneumonia and meningitis. Therefore, in this paper we are interested in filling this gap.

\section{Description of the model}

In this model we consider heterogeneous population. In this model we consider deterministic seven compartmental human population. The total population is divided into seven sub-classes, which are susceptible population $(S)$, pneumonia infectious $\left(I_{\mathrm{p}}\right)$, meningitis infectious $\left(I_{\mathrm{m}}\right)$, pneumonia and meningitis co-infectious $\left(I_{\mathrm{pm}}\right)$, pneumonia recovered $\left(R_{\mathrm{p}}\right)$, meningitis recovered $\left(R_{\mathrm{m}}\right)$, and pneumonia-meningitis co-infectious recovered $\left(R_{\mathrm{pm}}\right)$. Susceptible are recruited with rate of $\pi$ through birth or immigration, and their number increases from individuals that come from sub-classes of pneumonia recovered, meningitis recovered, and co-infectious recovered by losing their temporary immunity with rate of $\delta_{1}, \delta_{2}$, and $\delta_{3}$, respectively. In the entire susceptible population, individuals can get pneumonia with contact rate of $a$ from a pneumonia only infected or co-infected person with force of infection of pneumonia $f_{1}=\frac{a\left(I_{\mathrm{p}}+I_{\mathrm{pm}}\right)}{N}$ and join $I_{\mathrm{p}}$ compartment. In a similar way, individuals can get meningitis by contact rate of $b$ from a meningitis only infected or co-infected person with force of infection of meningitis $f_{2}=\frac{b\left(I_{\mathrm{m}}+I_{\mathrm{pm}}\right)}{N}$ and join $I_{\mathrm{m}}$ compartment. Pneumonia only infected individuals also can get an additional meningitis infection with force of infection $f_{2}$ and join co-infected compartment $\left(I_{\mathrm{pm}}\right)$. The coinfected compartment increases because of individuals that come from meningitis only infected compartment when they are infected by pneumonia with $f_{1}$ force of infection. Pneumonia only infected individuals can recover with rate of $\sigma_{1}$ and join pneumonia only recovered compartment $\left(I_{\mathrm{p}}\right)$. In a similar way, meningitis only infected individuals also recover with rate of $\sigma_{2}$ and join meningitis only recovered compartment $\left(I_{\mathrm{pm}}\right)$. The coinfected compartment also recovers with $\sigma$ rate, but those individuals either recover only from pneumonia disease and join pneumonia only recovered compartment with probability of $\sigma(1-e)$, or recover only from meningitis only disease and join meningitis only recovered compartment with probability of $\sigma g(1-e)$, or recover from both diseases and join co-infected recover compartment with probability of $\sigma(1-g)(1-e)$. In all compartments there is a natural death rate, which is $\mu$. Moreover, $\alpha_{1}$ is pneumonia only caused death rate, and $\alpha_{2}$ is meningitis caused death rate. The above description of the model is plotted in Fig. 1. From the flow graph (Fig. 1) of the model, we get the following system of differential:

$$
\left\{\begin{array}{l}
\frac{d S}{d t}=\pi+\delta_{1} R_{\mathrm{p}}+\delta_{2} R_{\mathrm{m}}+\delta_{3} R_{\mathrm{pm}}-\left(f_{1}+f_{2}+\mu\right) S \\
\frac{d I_{\mathrm{p}}}{d t}=f_{1} S-\left(f_{2}+\sigma_{1}+\alpha_{1}+\mu\right) I_{\mathrm{p}} \\
\frac{d I_{\mathrm{m}}}{d t}=f_{2} S-\left(f_{1}+\sigma_{2}+\alpha_{2}+\mu\right) I_{\mathrm{m}} \\
\frac{d I_{\mathrm{pm}}}{d t}=f_{2} I_{\mathrm{p}}+f_{1} I_{\mathrm{m}}-\left(\sigma+\alpha_{1}+\alpha_{2}+\mu\right) I_{\mathrm{pm}} \\
\frac{d R_{\mathrm{p}}}{d t}=\sigma_{1} I_{\mathrm{p}}+\sigma e I_{\mathrm{pm}}-\left(\delta_{1}+\mu\right) R_{\mathrm{p}} \\
\frac{d R_{\mathrm{m}}}{d t}=\sigma_{2} I_{\mathrm{m}}+\sigma g(1-e) I_{\mathrm{pm}}-\left(\delta_{2}+\mu\right) R_{\mathrm{m}} \\
\frac{d R_{\mathrm{pm}}}{d t}=\sigma(1-g)(1-e) I_{\mathrm{pm}}-\left(\delta_{3}+\mu\right) R_{\mathrm{pm}}
\end{array}\right.
$$

\section{Qualitative analysis}

In this section we study the qualitative behavior of the model. For simplification of the work, we split the full model into sub-models, which are pneumonia and meningitis only 
Figure 1 Flow diagram of the model

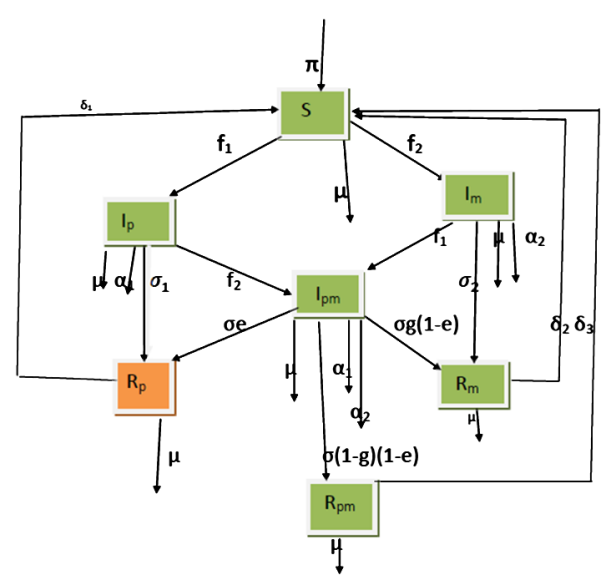

models. The qualitative behavior of the sub-model is studied first and that of the full model then follows.

\subsection{Pneumonia only model}

To get this model from equation (1), we set $I_{\mathrm{m}}=I_{\mathrm{pm}}=R_{\mathrm{m}}=R_{\mathrm{pm}}=0, \delta_{2}=\delta_{3}=f_{2}=0$, and then we get

$$
\left\{\begin{array}{l}
\frac{d S}{d t}=\pi+\delta_{1} R_{\mathrm{p}}-\left(f_{1}+\mu\right) S, \\
\frac{d I_{\mathrm{p}}}{d t}=f_{1} S-\left(\sigma_{1}+\alpha_{1}+\mu\right) I_{\mathrm{p}}, \\
\frac{d R_{\mathrm{p}}}{d t}=\sigma_{1} I_{\mathrm{p}}-\left(\delta_{1}+\mu\right) R_{\mathrm{p}} .
\end{array}\right.
$$

\subsubsection{Invariant region}

To get an invariant region, which shows as the solution is bounded, the total population of the model is $N=S+I_{\mathrm{p}}+R_{\mathrm{p}}$. By differentiating $N$ both sides and substituting respective expressions of $\frac{d S}{d t}, \frac{d I_{\mathrm{p}}}{d t}$, and $\frac{d R_{\mathrm{p}}}{d t}$ from equation (2), we get

$$
\frac{d N}{d t}=\pi+\mu N-\alpha_{1} I_{\mathrm{p}}
$$

If there is no death from pneumonia, equation (3) becomes

$$
\frac{d N}{d t} \leq \pi+\mu N
$$

Solving equation (4) gives

$$
\Omega=\left\{\left(S, I_{\mathrm{p}}, R_{\mathrm{p}}\right) \in \Re_{+}^{3}: 0 \leq N \leq \frac{\pi}{\mu} .\right.
$$

Therefore, all the solution set of (2) is bounded in $\Omega$.

\subsubsection{Positivity of the solution}

To show solutions of the model, as it is positive, first we take $\frac{d S}{d t}$ from equation (2).

$$
\frac{d S}{d t}=\pi+\delta_{1} R_{\mathrm{p}}-\left(f_{1}+\mu\right) S .
$$


We re-write equation (5) as

$$
\frac{d S}{d t} \geq-\left(f_{1}+\mu\right) t
$$

After evaluating equation (6), we obtain

$$
S \geq S_{0} e^{-\left(f_{1}+\mu\right) t}
$$

Similarly, we obtain

$$
\begin{aligned}
& I_{\mathrm{p}} \geq I_{p 0} e^{-\left(\sigma+\alpha_{1}+\mu\right) t}, \\
& R_{\mathrm{p}} \geq R_{p 0} e^{\left(\delta_{1}+\mu\right) R_{\mathrm{p}}} .
\end{aligned}
$$

Therefore, the solution of the model is positive for future time.

\subsubsection{Disease-free equilibrium (DFE)}

By equating equation (2) to zero and substituting $I_{\mathrm{p}}=0$, we obtain the disease-free equilibrium (DFE), $E_{0 p}=\left(\frac{\pi}{\mu}, 0,0\right)$.

\subsubsection{Basic reproduction number $\left(\Re_{0} p\right)$}

The basic reproduction number is an average number of secondary case infections by a single infected person in total susceptible population. To obtain $\Re_{0 p}$, we use the next generation matrix method that was formulated by [12], and we get

$$
\Re_{0 p}=\frac{a \pi}{\mu\left(\sigma_{1}+\alpha_{1}+\mu\right)} .
$$

\subsubsection{Local stability of DFE}

Theorem 1 The DFE is locally asymptotically stable if $\Re_{0 p}<1$ and unstable if $\Re_{0 p}>1$.

Proof To prove Theorem 1, first we take the second equation of equation (2) and consider the right-hand side $f=f_{1} S-\left(\sigma_{1}+\alpha_{1}+\mu\right) I_{\mathrm{p}}$. Then the partial derivative of $f$ with respect to $I_{\mathrm{p}}$ at disease-free equilibrium is

$$
\frac{\partial f}{\partial I_{\mathrm{p}}}\left(\frac{\pi}{\mu}, 0,0\right)=\frac{a \pi}{\mu}-\left(\sigma_{1}+\alpha_{1}+\mu\right) .
$$

For the disease-free equilibrium to be stable,

$$
\begin{aligned}
& \frac{\partial f}{\partial I_{\mathrm{p}}}\left(\frac{\pi}{\mu}, 0,0\right)=\frac{a \pi}{\mu}-\left(\sigma_{1}+\alpha_{1}+\mu\right)<0 \\
& \Rightarrow \quad\left(\sigma_{1}+\alpha_{1}+\mu\right)\left(\frac{a \pi}{\mu\left(\sigma_{1}+\alpha_{1}+\mu\right)}-1\right)<0 \\
& \Rightarrow \quad\left(\sigma_{1}+\alpha_{1}+\mu\right)\left(\Re_{0 p}-1\right)<0 .
\end{aligned}
$$

Therefore, the disease-free equilibrium is locally asymptomatically stable if $\Re_{0 p}<1$ and unstable otherwise. 


\subsubsection{Global stability of DFE}

Theorem 2 The DFE is globally asymptotically stable if $\Re_{0 p}<1$.

Proof To prove the global asymptotic stability of the DFE, we use the method of Lyapunov functions.

Systematically, we define a Lyapunov function $L$ such that

$$
L=\frac{1}{\left(\sigma_{1}+\alpha_{1}+\mu\right)} I_{\mathrm{p}}
$$

Then

$$
\begin{aligned}
\frac{d L}{d t} & =\frac{1}{\left(\sigma_{1}+\alpha_{1}+\mu\right)} \frac{d I_{\mathrm{p}}}{d t}=\frac{1}{\left(\sigma_{1}+\alpha_{1}+\mu\right)}\left(a S I_{\mathrm{p}}-\left(\sigma_{1}+\alpha_{1}+\mu\right) I_{\mathrm{p}}\right) \\
& \Rightarrow \frac{d L}{d t}=\frac{a S I_{\mathrm{p}}}{\left(\sigma_{1}+\alpha_{1}+\mu\right)}-I_{\mathrm{p}} \\
& \Rightarrow \frac{d L}{d t} \leq\left(\frac{a \pi}{\mu\left(\sigma_{1}+\alpha_{1}+\mu\right)}-1\right) I_{\mathrm{p}} \\
& \Rightarrow \frac{d L}{d t} \leq\left(\Re_{0 p}-1\right) I_{\mathrm{p}} .
\end{aligned}
$$

So $\frac{d L}{d t} \leq 0$ if $\Re_{0 p} \leq 1$. Furthermore, $\frac{d L}{d t}=0$ if $I_{\mathrm{p}}=0$ or $\Re_{0 p}=1$.

From this we see that $\left(S_{0}, 0,0\right)$ is the only singleton in $\left\{\left(S, I_{\mathrm{p}}, R_{\mathrm{p}}\right) \in \Omega_{1}: \frac{d L}{d t}=0\right\}$.

Therefore, by the principle of [12], DFE is globally asymptotically stable if $\Re_{0 p} \leq 1$.

\subsubsection{Endemic equilibrium (EE)}

The endemic equilibrium is denoted by $E_{\mathrm{p}}^{*}=\left(S^{*}, I_{\mathrm{p}}^{*}, R_{\mathrm{p}}^{*}\right)$ and it occurs when the disease persists in the community. To obtain it, we equate all the model equations (2) to zero. Then we obtain

$$
\begin{aligned}
S^{*} & =\frac{\pi}{\mu \Re_{0 p}}, \\
I_{\mathrm{p}}^{*} & =\frac{\left(\sigma_{1}+\mu+\alpha_{1}\right)\left(\delta_{1}+\mu\right)\left(\Re_{0} p-1\right)}{\delta_{1}\left(\alpha_{1}+\mu\right)+\mu\left(\sigma_{1}+\mu+\alpha_{1}\right)}, \\
R_{\mathrm{p}}^{*} & =\frac{\sigma_{1}}{\mu+\delta_{1}} I_{\mathrm{p}}^{*} .
\end{aligned}
$$

From this we see that for the endemic equilibrium to exist, $\Re_{0 p}>1$.

\subsubsection{Global stability of EE}

Theorem 3 If $\Re_{0 p}>1$, the EE (E*) of model (2) is globally asymptotically stable.

Proof Systematically, we define an appropriate Lyapunov function $L$ such that

$$
L=\left(S-S^{*}+S^{*} \ln \frac{S^{*}}{S}\right)+\left(I_{\mathrm{p}}-I_{\mathrm{p}}^{*}+I_{\mathrm{p}}^{*} \ln \frac{I_{\mathrm{p}}^{*}}{I_{\mathrm{p}}}\right)+\left(R_{P}-R_{P}^{*}+R_{\mathrm{p}}^{*} \ln \frac{R_{\mathrm{p}}^{*}}{R_{\mathrm{p}}}\right) .
$$

Then differentiating equation (8) with respect to $t$ gives

$$
\frac{d L}{d t}=\left(\frac{S-S^{*}}{S}\right) \frac{d S}{d t}+\left(\frac{I_{\mathrm{p}}-I_{\mathrm{p}}^{*}}{I_{\mathrm{p}}}\right) \frac{d I_{\mathrm{p}}}{d t}+\left(\frac{R_{\mathrm{p}}-R_{\mathrm{p}}^{*}}{R_{\mathrm{p}}}\right) \frac{d R_{\mathrm{p}}}{d t} .
$$


After substituting expressions for $\frac{d S}{d t}, \frac{d I_{\mathrm{p}}}{d t}$, and $\frac{d R_{\mathrm{p}}}{d t}$ from (2) into (8) and collecting all positive terms together and also negative terms together, we obtain

$$
\frac{d L}{d t}=L_{1}-L_{2}
$$

where $L_{1}=\pi+\delta_{1} R_{\mathrm{p}}+f_{1} S+\sigma_{1} I_{\mathrm{p}}+\frac{\delta_{1} R_{\mathrm{p}}^{*} S^{*}}{S}+\frac{f_{1} S^{*} I_{\mathrm{p}}^{*}}{I_{\mathrm{p}}}+\frac{\sigma_{1} I_{\mathrm{p}}^{*} R_{\mathrm{p}}^{*}}{R_{\mathrm{p}}}, L_{2}=\delta_{1} R_{\mathrm{p}}^{*}+f_{1} S^{*}+f_{1} S I_{\mathrm{p}}^{*}+\frac{\pi S^{*}}{S}+$ $\frac{\delta_{1} R_{\mathrm{p}} S^{*}}{S}+\frac{\left(f_{1}+\mu\right)\left(S-S^{*}\right)^{2}}{S}+\frac{f_{1} S I_{\mathrm{p}}^{*}}{I_{\mathrm{p}}}+\frac{\left(\sigma_{1}+\alpha_{1}+\mu\right)\left(I_{\mathrm{p}}-I_{\mathrm{p}}^{*}\right)^{2}}{I_{\mathrm{p}}}+\frac{\sigma_{1} I_{\mathrm{p}} R_{\mathrm{p}}^{*}}{R_{\mathrm{p}}}+\frac{\left(\delta_{1}+\mu\right)\left(R_{\mathrm{p}}-R_{\mathrm{p}}^{*}\right)^{2}}{R_{\mathrm{p}}}$.

Thus if $L_{1}<L_{2}$, then $\frac{d L}{d t} \leq 0$ and $\frac{d L}{d t}=0$ if and only if $S=S^{*}, I_{\mathrm{p}}=I_{P}^{*}, R_{P}=R_{\mathrm{p}}^{*}$.

From this, we see that $E^{*}=\left(S^{*}, I_{P}^{*}, R_{\mathrm{p}}^{*}\right)$ is the largest compact invariant singleton set in $\left.\left\{\left(S^{*}, I_{P}^{*}, R_{\mathrm{p}}^{*}\right)\right) \in \Omega_{1}: \frac{d L}{d t}=0\right\}$. Therefore, by the principle of [13], the endemic equilibrium $\left(E^{*}\right)$ is globally asymptotically stable in the invariant region if $L_{1}<L_{2}$.

\subsection{Meningitis only model}

By letting $I_{P}=I_{\mathrm{pm}}=R_{\mathrm{p}}=R_{\mathrm{pm}}=0$ in equation (1), we obtain the meningitis only model.

$$
\left\{\begin{array}{l}
\frac{d S}{d t}=\pi+\delta_{2} R_{t}-\left(f_{2}+\mu\right) S \\
\frac{d I_{\mathrm{m}}}{d t}=f_{2} S-\left(\sigma_{2}+\alpha_{2}+\mu\right) I_{\mathrm{m}} \\
\frac{d R_{\mathrm{m}}}{d t}=\sigma_{2} I_{\mathrm{m}}-\left(\delta_{2}+\mu\right) R_{\mathrm{m}} .
\end{array}\right.
$$

\subsubsection{Invariant region}

In this section we obtain a region in which the solution of (10) is bounded. For this model, the total human population $N_{2}=S+I_{\mathrm{m}}+R_{\mathrm{m}}$. Then, after differentiating $N_{2}$ with respect to time and substituting the expression for $\frac{d S}{d t}, \frac{d I_{\mathrm{m}}}{d t}$, and $\frac{d R_{\mathrm{m}}}{d t}$ from equation (10), we obtain

$$
\frac{d N_{2}}{d t}=\pi-\mu N_{2}-\alpha_{2} I_{\mathrm{m}}
$$

If there is no death from meningitis, equation (11) becomes

$$
\frac{d N_{2}}{d t} \leq \pi-\mu N_{2}
$$

After solving equation (12) and evaluating it as time tends to infinity, we get

$$
\Omega_{2}=\left\{\left(S, I_{\mathrm{m}}, R_{\mathrm{m}}\right) \in \mathfrak{R}_{+}^{3}: 0 \leq N_{2} \leq \frac{\pi}{\mu}\right\} .
$$

Therefore, all the solution set of (10) is bounded in $\Omega_{2}$.

\subsubsection{Positivity of the solution}

In this section we show that all the solutions of model (10) are positive for future time.

We take $\frac{d S}{d t}$ from equation (10),

$$
\frac{d S}{d t}=\pi+\delta_{2} R_{\mathrm{m}}-f_{2} S-\mu S
$$

It is true that equation (13) can be written as

$$
\frac{d S}{d t} \geq-(\mu) S \text {. }
$$


After solving equation (14), we get

$$
S \geq S_{0} e^{-(\mu) t}
$$

Similarly, we obtain

$$
\begin{aligned}
& I_{\mathrm{m}} \geq I_{m_{0}} e^{-\left(\sigma_{2}+\alpha_{2}+\mu\right) t}, \\
& R_{\mathrm{m}} \geq R_{m_{0}} e^{\left(\delta_{2}+\mu\right) R_{\mathrm{m}}} .
\end{aligned}
$$

\subsubsection{Disease-free equilibrium (DFE)}

By letting equation (10) to zero and evaluating at $I_{\mathrm{m}}=0$, we get DFE $E_{02}=\left(\frac{\pi}{\mu}, 0,0,0\right)$.

\subsubsection{Basic reproduction number $\left(\Re_{0 m}\right)$}

The basic reproduction number is an average number of secondary case infections by a single infected person in total susceptible population. To obtain $\mathfrak{R}_{0 m}$, we use the next generation matrix method that was formulated by [12]. Then we get

$$
\Re_{0 m}=\frac{b \pi}{\mu\left(\sigma_{2}+\alpha_{2}+\mu\right)} .
$$

\subsubsection{Local stability of DFE}

Theorem 4 The DFE point is locally asymptotically stable if $\Re_{0 m}<1$ and unstable if $\Re_{0 m}>1$.

Proof To prove this, let us take the right-hand side expression of the second equation of (10) $f=f_{2} S-\left(\sigma_{2}+\alpha_{2}+\mu\right) I_{\mathrm{m}}$. Then the partial derivative of $f$ with respect to $I_{\mathrm{m}}$ at the disease-free equilibrium is

$$
\frac{\partial f}{\partial I_{\mathrm{m}}}\left(\frac{\pi}{\mu}, 0,0\right)=\frac{b \pi}{\mu}-\left(\sigma_{2}+\alpha_{2}+\mu\right) .
$$

For the disease-free equilibrium to be stable,

$$
\begin{aligned}
& \frac{\partial f}{\partial I_{\mathrm{m}}}\left(\frac{\pi}{\mu}, 0,0\right)=\frac{b \pi}{\mu}-\left(\sigma_{2}+\alpha_{2}+\mu\right)<0 \\
& \Rightarrow \quad\left(\sigma_{2}+\alpha_{2}+\mu\right)\left(\frac{b \pi}{\mu\left(\sigma_{2}+\alpha_{2}+\mu\right)}-1\right)<0 \\
& \Rightarrow \quad\left(\sigma_{2}+\alpha_{2}+\mu\right)\left(\Re_{0 m}-1\right)<0 .
\end{aligned}
$$

Therefore, the disease-free equilibrium is locally asymptomatically stable if $\Re_{0 m}<1$ and unstable otherwise.

\subsubsection{Global stability of DFE}

Theorem 5 The DFE is globally asymptotically stable if $\Re_{0 m}<1$.

Proof To prove the global asymptotic stability of the DFE, we use the method of Lyapunov functions. 
Systematically, we define a Lyapunov function $L$ such that

$$
M=\frac{1}{\left(\sigma_{2}+\alpha_{2}+\mu\right)} I_{\mathrm{m}}
$$

Then

$$
\begin{aligned}
& \frac{d M}{d t}=\frac{1}{\left(\sigma_{2}+\alpha_{2}+\mu\right)} \frac{d I_{\mathrm{m}}}{d t}=\frac{1}{\left(\sigma_{2}+\alpha_{2}+\mu\right)}\left(b S I_{\mathrm{m}}-\left(\sigma_{2}+\alpha_{2}+\mu\right) I_{\mathrm{m}}\right) \\
& \Rightarrow \quad \frac{d M}{d t}=\frac{b S I_{\mathrm{m}}}{\left(\sigma_{2}+\alpha_{2}+\mu\right)}-I_{\mathrm{m}} \\
& \Rightarrow \quad \frac{d M}{d t} \leq\left(\frac{b \pi}{\mu\left(\sigma_{2}+\alpha_{2}+\mu\right)}-1\right) I_{\mathrm{m}} \\
& \Rightarrow \quad \frac{d L}{d t} \leq\left(\Re_{0 m}-1\right) I_{\mathrm{m}} .
\end{aligned}
$$

So, $\frac{d M}{d t} \leq 0$ if $\Re_{0 m} \leq 1$. Furthermore, $\frac{d M}{d t}=0$ if $I_{\mathrm{m}}=0$ or $\Re_{0 m}=1$.

From this we see that $\left(S_{0}, 0,0\right)$ is the only singleton in $\left\{\left(S, I_{\mathrm{m}}, R_{\mathrm{m}}\right) \in \Omega_{2}: \frac{d M}{d t}=0\right\}$.

Therefore, by the principle of [12], DFE is globally asymptotically stable if $\Re_{0 m} \leq 1$.

\subsubsection{Endemic equilibrium (EE)}

The endemic equilibrium is denoted by $E_{\mathrm{m}}^{*}=\left(S^{*}, I_{\mathrm{m}}^{*}, R_{\mathrm{m}}^{*}\right)$ and it occurs when the disease persists in the community. To obtain it, we equate all the model equations (10) to zero. Then we obtain

$$
\begin{aligned}
& S^{*}=\frac{\pi}{\mu \Re_{0 m}}, \\
& I_{\mathrm{m}}^{*}=\frac{\left(\sigma_{2}+\mu+\alpha_{2}\right)\left(\delta_{2}+\mu\right)\left(\Re_{0} m-1\right)}{\delta_{1}\left(\alpha_{1}+\mu\right)+\mu\left(\sigma_{1}+\mu+\alpha_{1}\right)}, \\
& R_{\mathrm{m}}^{*}=\frac{\sigma_{2}}{\mu+\delta_{2}} I_{\mathrm{m}}^{*} .
\end{aligned}
$$

From this we see that for the endemic equilibrium to exist, $\Re_{0 m}>1$.

Lemma 6 A unique endemic equilibrium point $E^{*}$ exists and is positive if $\Re_{0 m}>1$.

\subsubsection{Global stability of EE}

Theorem 7 If $\Re_{0 m}>1$, the EE (E*) of model (10) is globally asymptotically stable.

Proof Systematically, we define an appropriate Lyapunov function $G$ such that

$$
G=\left(S-S^{*}+S^{*} \ln \frac{S^{*}}{S}\right)+\left(I_{\mathrm{m}}-I_{\mathrm{m}}^{*}+I_{\mathrm{m}}^{*} \ln \frac{I_{\mathrm{m}}^{*}}{I_{\mathrm{m}}}\right)+\left(R_{\mathrm{m}}-R_{\mathrm{m}}^{*}+R_{\mathrm{m}}^{*} \ln \frac{R_{\mathrm{m}}^{*}}{R_{\mathrm{m}}}\right) .
$$

Then differentiating equation (16) with respect to $t$ gives

$$
\frac{d G}{d t}=\left(\frac{S-S^{*}}{S}\right) \frac{d S}{d t}+\left(\frac{I_{\mathrm{m}}-I_{\mathrm{m}}^{*}}{I_{\mathrm{m}}}\right) \frac{d I_{\mathrm{m}}}{d t}+\left(\frac{R_{\mathrm{m}}-R_{\mathrm{m}}^{*}}{R_{\mathrm{m}}}\right) \frac{d R_{\mathrm{m}}}{d t} .
$$


After substituting expressions for $\frac{d S}{d t}, \frac{d I_{\mathrm{m}}}{d t}$, and $\frac{d R_{\mathrm{m}}}{d t}$ from (10) into (16) and collecting all positive terms together and also negative terms together, we obtain

$$
\frac{d G}{d t}=G_{1}-G_{2}
$$

where $G_{1}=\pi+\delta_{2} R_{\mathrm{m}}+f_{2} S+\sigma_{2} I_{\mathrm{m}}+\frac{\delta_{2} R_{\mathrm{m}}^{*} S^{*}}{S}+\frac{f_{2} S^{*} I_{\mathrm{m}}^{*}}{I_{\mathrm{m}}}+\frac{\sigma_{2} I_{\mathrm{m}}^{*} R_{\mathrm{m}}^{*}}{R_{\mathrm{m}}}, G_{2}=\delta_{2} R_{\mathrm{m}}^{*}+f_{2} S^{*}+f_{2} S I_{\mathrm{m}}^{*}+\frac{\pi S^{*}}{S}+$ $\frac{\delta_{2} R_{\mathrm{m}} S^{*}}{S}+\frac{\left(f_{2}+\mu\right)\left(S-S^{*}\right)^{2}}{S}+\frac{f_{2} S_{\mathrm{m}}^{*}}{I_{\mathrm{m}}}+\frac{\left(\sigma_{2}+\alpha_{2}+\mu\right)\left(I_{\mathrm{m}}-I_{\mathrm{m}}^{*}\right)^{2}}{I_{\mathrm{m}}}+\frac{\sigma_{2} I_{\mathrm{m}} R_{\mathrm{m}}^{*}}{R_{\mathrm{m}}}+\frac{\left(\delta_{2}+\mu\right)\left(R_{\mathrm{m}}-R_{\mathrm{m}}^{*}\right)^{2}}{R_{\mathrm{m}}}$.

Thus if $G_{1}<L_{G}$, then $\frac{d G}{d t} \leq 0$ and $\frac{d G}{d t}=0$ if and only if $S=S^{*}, I_{\mathrm{m}}=I_{\mathrm{m}}^{*}, R_{\mathrm{m}}=R_{\mathrm{m}}^{*}$.

From this, we see that $E^{*}=\left(S^{*}, I_{\mathrm{m}}^{*}, R_{\mathrm{m}}^{*}\right)$ is the largest compact invariant singleton set in $\left.\left\{\left(S^{*}, I_{\mathrm{m}}^{*}, R_{\mathrm{m}}^{*}\right)\right) \in \Omega_{2}: \frac{d G}{d t}=0\right\}$. Therefore, by the principle of [13], the endemic equilibrium $\left(E^{*}\right)$ is globally asymptotically stable in the invariant region if $G_{1}<G_{2}$.

\subsection{Pneumonia-meningitis co-infection model}

The model equation of pneumonia and meningitis co-infection given in equation (1) is as follows:

$$
\left\{\begin{array}{l}
\frac{d S}{d t}=\pi+\delta_{1} R_{\mathrm{p}}+\delta_{2} R_{\mathrm{m}}+\delta_{3} R_{\mathrm{pm}}-\left(f_{1}+f_{2}+\mu\right) S \\
\frac{d I_{\mathrm{p}}}{d t}=f_{1} S-\left(f_{2}+\sigma_{1}+\alpha_{1}+\mu\right) I_{\mathrm{p}} \\
\frac{d I_{\mathrm{m}}}{d t}=f_{2} S-\left(f_{1}+\sigma_{2}+\alpha_{2}+\mu\right) I_{\mathrm{m}} \\
\frac{d I_{\mathrm{pm}}}{d t}=f_{2} I_{\mathrm{p}}+f_{1} I_{\mathrm{m}}-\left(\sigma+\alpha_{1}+\alpha_{2}+\mu\right) I_{\mathrm{pm}} \\
\frac{d R_{\mathrm{p}}}{d t}=\sigma_{1} I_{\mathrm{p}}+\sigma e I_{\mathrm{pm}}-\left(\delta_{1}+\mu\right) R_{\mathrm{p}} \\
\frac{d R_{\mathrm{m}}}{d t}=\sigma_{2} I_{\mathrm{m}}+\sigma g(1-e) I_{\mathrm{pm}}-\left(\delta_{2}+\mu\right) R_{\mathrm{m}} \\
\frac{d R_{\mathrm{pm}}}{d t}=\sigma(1-g)(1-e) I_{\mathrm{pm}}-\left(\delta_{3}+\mu\right) R_{\mathrm{pm}}
\end{array}\right.
$$

where $f_{1}=\frac{a\left(I_{\mathrm{p}}+I_{\mathrm{pm}}\right)}{N}, f_{2}=\frac{b\left(I_{\mathrm{m}}+I_{\mathrm{pm}}\right)}{N}$, and $S(0)=S_{0}, I_{\mathrm{p}}(0)=I_{p_{0}}, I_{\mathrm{m}}(0)=I_{m_{0}}, I_{\mathrm{pm}}(0)=I_{p m_{0}}$, $R_{\mathrm{p}}(0)=R_{p 0}, R_{\mathrm{m}}(0)=R_{m 0}, R_{\mathrm{pm}}(0)=R_{p m 0}$, and $B(0)=B_{0}$ are non-negative initial values.

\subsection{Qualitative analysis}

\subsubsection{Invariant region}

To obtain invariant region, we consider the total population, which is $N=S+I_{\mathrm{p}}+I_{\mathrm{m}}+$ $I_{\mathrm{pm}}+R_{\mathrm{p}}+R_{\mathrm{m}}+R_{\mathrm{pm}}$. Then, by a technique similar to previous sections, we obtain

$$
\frac{d N}{d t}=\pi-\mu N-\alpha_{1}\left(I_{\mathrm{p}}+I_{\mathrm{pm}}\right)-\alpha_{2}\left(I_{\mathrm{m}}+I_{\mathrm{pm}}\right) .
$$

If we do not consider deaths from pneumonia and meningitis, then (19) becomes

$$
\frac{d N}{d t} \leq \pi-\mu N
$$

Then solving equation (20) gives

$$
\Omega=\left\{\left(S, I_{\mathrm{p}}, I_{\mathrm{m}}, I_{\mathrm{pm}}, R_{\mathrm{p}}, R_{\mathrm{m}}, R_{\mathrm{pm}}\right) \in \Re^{7}: 0 \leq N \leq \frac{\pi}{\mu}\right\} .
$$




\subsubsection{Positivity of the solution}

Theorem 8 If $S_{0}>0, I_{p_{0}}>0, I_{m_{0}}>0, I_{p m_{0}}>0, R_{p 0}>0, R_{m 0}>0, R_{p m 0}>0$, then all the solution set $\left(S(t), I_{\mathrm{p}}(t), I_{\mathrm{m}}(t), I_{\mathrm{pm}}(t), R_{\mathrm{p}}(t), R_{\mathrm{m}}(t), R_{\mathrm{pm}}(t)\right)$ is positive for future time.

Proof Consider $t_{1}$ defined as follows:

$$
\begin{aligned}
t_{1}= & \sup \left\{t>0: S(\tau)>0, I_{\mathrm{p}}(\tau)>0, I_{\mathrm{m}}(\tau)>0, I_{\mathrm{pm}}(\tau)>0, R_{\mathrm{p}}(\tau)>0, R_{\mathrm{m}}(\tau)>0, R_{\mathrm{pm}}(\tau)>0,\right. \\
& \text { for all } \tau \in[0, t]\} .
\end{aligned}
$$

Since $S_{0} \geq 0, I_{p 0} \geq 0, I_{m_{0}} \geq 0, I_{p m_{0}} \geq 0, R_{p 0} \geq 0, R_{m 0} \geq 0, R_{p m 0} \geq 0$, thus $t_{1}>0$. If $t_{1}<\infty$, then necessarily $S$ or $I_{\mathrm{p}}$ or $I_{\mathrm{m}}$ or $I_{\mathrm{pm}}$ or $R_{\mathrm{p}}$ or $R_{\mathrm{m}}$ or $R_{\mathrm{pm}}$ is equal to zero at $t_{1}$. From equation (18), let us take the first equation

$$
\frac{d S}{d t}=\pi+\delta_{1} R_{\mathrm{p}}+\delta_{2} R_{\mathrm{m}}+\delta_{3} R_{\mathrm{pm}}-\left(f_{1}+f_{2}+\mu\right) S .
$$

Using the variation of constants formula, the solution of equation (21) at $t_{1}$ is given by

$$
\begin{aligned}
S\left(t_{1}\right)= & S(0) \exp \left[-\int_{0}^{t_{1}}\left(f_{1}+f_{2}+\mu\right)(s) d s\right] \\
& +\int_{0}^{t_{1}}\left(\pi+\delta_{1} R_{\mathrm{p}}+\delta_{2} R_{\mathrm{m}}+\delta_{3} R_{\mathrm{pm}}\right) \exp \left[-\int_{s}^{t_{1}}\left(f_{1}+f_{2}+\mu\right)(\tau) d \tau\right] d s .
\end{aligned}
$$

Moreover, since all the variables are positive in $\left[0, t_{1}\right]$, then $S\left(t_{1}\right)>0$.

It can be shown in a similar way that $I_{\mathrm{p}}\left(t_{1}\right)>0, I_{\mathrm{m}}\left(t_{1}\right)>0, I_{\mathrm{pm}}\left(t_{1}\right)>0, R_{\mathrm{p}}\left(t_{1}\right)>0, R_{\mathrm{m}}\left(t_{1}\right)>$ 0 , and $R_{\mathrm{pm}}\left(t_{1}\right)>0$, which is a contradiction. Hence $t_{1}=\infty$.

\subsubsection{Disease-free equilibrium}

By substituting $I_{\mathrm{p}}=0, I_{\mathrm{m}}=0$, and $I_{\mathrm{pm}}=0$ in equation (18), DFE becomes

$$
E_{0}=\left(\frac{\pi}{\mu}, 0,0,0,0,0,0\right)
$$

\subsubsection{Basic reproduction number $\left(\Re_{0}\right)$}

Let us consider the infective compartments of the model

$$
\begin{aligned}
& \frac{d I_{\mathrm{p}}}{d t}=f_{1} S-\left(f_{1}+\sigma_{1}+\alpha_{1}+\mu\right) I_{\mathrm{p}} \\
& \frac{d I_{\mathrm{m}}}{d t}=f_{2} S-\left(f_{1}+\sigma_{2}+\alpha_{2}+\mu\right) I_{\mathrm{m}} \\
& \frac{d I_{\mathrm{pm}}}{d t}=f_{2} I_{\mathrm{p}}+f_{1} I_{\mathrm{m}}-\left(\sigma+\alpha_{1}+\alpha_{2}+\mu\right) I_{\mathrm{pm}}
\end{aligned}
$$

By using the next generation matrix outlined in [12], we obtain $F$ and $V$ in the following way:

$$
F=\left(\begin{array}{ccc}
\frac{a \pi}{\mu} & 0 & \frac{a \pi}{\mu} \\
0 & \frac{b \pi}{\mu} & \frac{b \pi}{\mu} \\
0 & 0 & 0
\end{array}\right) \quad \& \quad V=\left(\begin{array}{ccc}
\left(\sigma_{1}+\alpha_{1}+\mu\right) & 0 & 0 \\
0 & \left(\sigma_{2}+\alpha_{2}+\mu\right) & 0 \\
0 & 0 & \left(\sigma+\alpha_{1}+\alpha_{2}+\mu\right)
\end{array}\right)
$$




$$
F V^{-1}=\left(\begin{array}{ccc}
\frac{a \pi}{\mu\left(\sigma_{1}+\alpha_{1}+\mu\right)} & 0 & \frac{a \pi}{\mu\left(\sigma+\alpha_{1}+\alpha_{2}+\mu\right)} \\
0 & \frac{b \pi}{\mu\left(\sigma_{2}+\alpha_{2}+\mu\right)} & \frac{b \pi}{\mu\left(\sigma+\alpha_{1}+\alpha_{2}+\mu\right)} \\
0 & 0 & 0
\end{array}\right) .
$$

The eigenvalues of $F V^{-1}$ are

$$
\begin{aligned}
& \lambda_{1}^{*}=\frac{a \pi}{\mu\left(\sigma_{1}+\alpha_{1}+\mu\right)}=\Re_{0 p}, \\
& \lambda_{2}^{*}=\frac{b \pi}{\mu\left(\sigma_{2}+\alpha_{2}+\mu\right)}=\Re_{0 m}, \\
& \lambda_{3}^{*}=0 .
\end{aligned}
$$

The dominant eigenvalue, which is the basic reproduction number of $F V^{-1}$, is

$$
\Re_{0}=\max \left\{\Re_{0 p}, \Re_{0 m}\right\} .
$$

\subsubsection{Local stability of disease-free equilibrium}

Theorem 9 The disease-free equilibrium point is locally asymptotically stable if $\Re_{0}<1$, otherwise unstable.

Proof The Jacobian matrix of the model at DFE is obtained below in equation (22).

$$
J_{E_{0}}=\left[\begin{array}{ccccccc}
-\mu & \frac{-a \pi}{\mu} & 0 & \frac{-b \pi}{\mu} & \delta_{1} & \delta_{2} & \delta_{3} \\
0 & \frac{-a \pi}{\mu}-k_{1} & 0 & \frac{-b \pi}{\mu} & 0 & 0 & 0 \\
0 & 0 & \frac{b \pi}{\mu}-k_{2} & \frac{b \pi}{\mu} & 0 & 0 & 0 \\
0 & 0 & 0 & -k_{3} & 0 & 0 & 0 \\
0 & \delta_{1} & 0 & \sigma e & -k_{4} & 0 & 0 \\
0 & 0 & \delta_{2} & \sigma g(1-e) & 0 & -k_{5} & 0 \\
0 & 0 & 0 & \sigma(1-g)(1-e) & 0 & 0 & -k_{6}
\end{array}\right] \text {, }
$$

where

$$
\begin{aligned}
& k_{1}=\sigma_{1}+\alpha_{1}+\mu, \\
& k_{2}=\sigma_{2}+\alpha_{2}+\mu, \\
& k_{3}=\sigma+\alpha_{1}+\alpha_{2}+\mu, \\
& k_{4}=\delta_{1}+\mu, \\
& k_{5}=\delta_{2}+\mu, \\
& k_{6}=\delta_{3}+\mu .
\end{aligned}
$$

From equation (22) we can get the following characteristic polynomial in equation (23).

$$
\begin{aligned}
p(\lambda) & =(-\mu-\lambda)\left(-k_{4}-\lambda\right)\left(-k_{5}-\lambda\right)\left(-k_{3}-\lambda\right)\left(-k_{6}-\lambda\right)\left(\frac{a \pi}{\mu}-k_{1}-\lambda\right)\left(\frac{b \pi}{\mu}-k_{2}-\lambda\right) \\
& =0 .
\end{aligned}
$$


Then from equation (23) we can get

$$
\begin{aligned}
& \lambda_{1}=-\mu<0, \\
& \lambda_{2}=-k_{4}<0, \\
& \lambda_{3}=-k_{5}<0, \\
& \lambda_{4}=-k_{3}<0, \\
& \lambda_{5}=-k_{6}<0, \\
& \lambda_{6}=\frac{a \pi}{\mu}-k_{1}, \\
& \lambda_{7}=\frac{b \pi}{\mu}-k_{2} .
\end{aligned}
$$

Therefore, the DFE to be stable, $\lambda_{6}$ and $\lambda_{7}$ must be negative, i.e., $\frac{a \pi}{\mu}-k_{1}<0$ and $\frac{b \pi}{\mu}-k_{2}<0$. For $\frac{a \pi}{\mu}-k_{1}<0$ means that $\frac{a \pi}{\mu}<k_{1}$, which gives us $\frac{a \pi}{\mu k_{1}}<1$, this means $\Re_{0 p}<1$.

Similarly, for $\frac{b \pi}{\mu}-k_{2}<0$ means that $\frac{b \pi}{\mu k_{2}}<1$, this is equivalent to $\Re_{0 m}<1$. Therefore, DFE is locally asymptotically stable if and only if $\Re_{0}=\operatorname{Max}\left\{\Re_{0 p}, \Re_{0 m}\right\}<1$.

\subsection{Global asymptotic stability of disease-free equilibrium}

To investigate the global stability of disease-free equilibrium, we use the technique implemented by [9]. First the full pneumonia-meningitis model (18) can be re-written as follows:

$$
\begin{aligned}
& \frac{d X}{d t}=F(X, Z), \\
& \frac{d Z}{d t}=G(X, Z), \quad G(X, 0)=0,
\end{aligned}
$$

where $X$ stands for the uninfected population, that is, $X=\left(S, R_{\mathrm{p}}, R_{\mathrm{m}}, R_{\mathrm{pm}}\right)$, and $Z$ also stands for the infected population, that is, $Z=\left(I_{\mathrm{p}}, I_{\mathrm{m}}, I_{\mathrm{pm}}\right)$. The disease-free equilibrium point of the model is denoted by $U=\left(X^{*}, 0\right)$.

The point $U=\left(X^{*}, 0\right)$ is a globally asymptotically stable equilibrium for the model provided that $\Re_{0}<1$ (which is locally asymptotically stable) and the following conditions must be met:

$\left(H_{1}\right)$ For $\frac{d X}{d t}=F(X, 0), X^{*}$ is globally asymptotically stable;

$\left(H_{2}\right) G(X, Z)=A Z-\tilde{G}(X, Z), \tilde{G}(X, Z) \geq 0$ for $(X, Z) \in \Omega$.

If model (18) meets the above two criteria, then the following theorem holds.

Theorem 10 The point $U=\left(X^{*}, 0\right)$ is globally asymptotically stable equilibrium provided that $\Re_{0}<1$ and conditions $\left(H_{1}\right)$ and $\left(H_{2}\right)$ are satisfied.

Proof From system (18) we can get $F(X, Z)$ and $G(X, Z)$ :

$$
F(X, Z)=\left(\begin{array}{c}
\pi+\delta_{1} R_{\mathrm{p}}+\delta_{2} R_{\mathrm{m}}+\delta_{3} R_{\mathrm{pm}}-\left(f_{1}+f_{2}+\mu\right) S \\
\sigma_{1} I_{\mathrm{p}}+\sigma e I_{\mathrm{pm}}-\left(\delta_{1}+\mu\right) R_{\mathrm{p}} \\
\sigma_{2} I_{\mathrm{m}}+\sigma g(1-e) I_{\mathrm{pm}}-\left(\delta_{2}+\mu\right) R_{\mathrm{m}} \\
\sigma(1-g)(1-e) I_{\mathrm{pm}}-\left(\delta_{3}+\mu\right) R_{\mathrm{pm}}
\end{array}\right),
$$




$$
G(X, Z)=\left(\begin{array}{c}
f_{1} S-\left(f_{2}+\sigma_{1}+\alpha_{1}+\mu\right) I_{\mathrm{p}} \\
f_{2} S-\left(f_{1}+\sigma_{2}+\alpha_{2}+\mu\right) I_{\mathrm{m}} \\
f_{2} I_{\mathrm{p}}+f_{1} I_{\mathrm{m}}-\left(\sigma+\alpha_{1}+\alpha_{2}+\mu\right) I_{\mathrm{pm}}
\end{array}\right)
$$

Consider the reduced system:

$$
\left.\frac{d X}{d t}\right|_{Z=0}=\left(\begin{array}{c}
\pi-\mu S \\
0 \\
0 \\
0
\end{array}\right) .
$$

From equation (24), it is obvious that $X^{*}=\left(\frac{\pi}{\mu}, 0\right)$ is the global asymptotic point. This can be verified from the solution, namely $S=\frac{\pi}{\mu}+\left(S(0)-\frac{\pi}{\mu}\right) e^{-\mu t}$. As $t \rightarrow \infty$, the solution $(S) \rightarrow \frac{\pi}{\mu}$ implies the global convergence of (24) in $\Omega$.

Let

$$
A=\left(\begin{array}{ccc}
a-\left(\sigma_{1}+\alpha_{1}+\mu\right) & 0 & a \\
0 & -\left(\sigma_{2}+\alpha_{2}+\mu\right) & 0 \\
0 & 0 & -\left(\sigma+\alpha_{1}+\alpha_{2}+\mu\right)
\end{array}\right) .
$$

Then $G(X, Z)$ can be written as $G(X, Z)=A Z-\tilde{G}(X, Z)$, where

$$
\tilde{G}(X, Z)=\left(\begin{array}{c}
\tilde{G}_{1}(X, Z) \\
\tilde{G}_{2}(X, Z) \\
\tilde{G}_{3}(X, Z)
\end{array}\right)=\left(\begin{array}{c}
a\left(I_{\mathrm{m}}+I_{\mathrm{pm}}\right)\left(1-\frac{S}{N}\right)+f_{2} I_{\mathrm{p}} \\
f_{1} I_{\mathrm{m}} \\
-\left(f_{2} I_{\mathrm{p}}+f_{1} I_{\mathrm{m}}\right.
\end{array}\right) .
$$

In equation (25), $\tilde{G}_{2}(X, Z)<0$, which leads to $\tilde{G}(X, Z)<0$; that means the second condition $\left(H_{2}\right)$ is not satisfied, so $U=\left(X^{*}, 0\right)$ may not be globally asymptotically stable when $\Re_{0}<1$.

\subsection{Sensitivity analysis}

In this subsection sensitivity analysis is performed to identify the most influential parameters for the expansion as well as for control of infection in the community. To perform this, we use techniques described in [14]. The sensitivity index of $\Re_{0}$ with respect to a parameter, say $x$, is given by $\Lambda_{x}^{\Re_{0}}=\frac{\partial \Re_{0}}{\partial x} \frac{x}{\Re_{0}}$. Since $\Re_{0}=\max \left\{\Re_{0 p}, \Re_{0 m}\right\}$, we obtain the sensitivity analysis of $\Re_{0 p}$ and $\Re_{0 m}$ separately in the following way:

$$
\begin{aligned}
& \Lambda_{a}^{\Re_{0 p}}=\frac{\partial \Re_{0 p}}{\partial a} \frac{a}{\Re_{0 p}}=\frac{\pi}{\mu\left(\sigma_{1}+\alpha_{1}+\mu\right)} \frac{a \mu\left(\sigma_{1}+\alpha_{1}+\mu\right)}{a \pi}=1>0, \\
& \Lambda_{\alpha_{1}}^{\Re_{0 p}}=\frac{\partial \Re_{0 p}}{\partial \alpha_{1}} \frac{\alpha_{1}}{\Re_{0 p}}=-\frac{\alpha_{1}}{\left(\sigma_{1}+\alpha_{1}+\mu\right)}<0, \\
& \Lambda_{\sigma_{1}}^{\Re_{0 p}}=\frac{\partial \Re_{0 p}}{\partial \sigma_{1}} \frac{\sigma_{1}}{\Re_{0 p}}=-\frac{\sigma_{1}}{\left(\sigma_{1}+\alpha_{1}+\mu\right)}<0, \\
& \Lambda_{\mu}^{\Re_{0 p}}=\frac{\partial \Re_{0 p}}{\partial \mu} \frac{\mu}{\Re_{0 p}}=-\frac{\left(\sigma_{1}+\alpha_{1}+2 \mu\right)}{\left(\sigma_{1}+\alpha_{1}+\mu\right)}<0, \\
& \Lambda_{b}^{\Re_{0 m}}=\frac{\partial \Re_{0 m}}{\partial b} \frac{b}{\Re_{0 m}}=\frac{\pi}{\mu\left(\sigma_{2}+\alpha_{2}+\mu\right)} \frac{b \mu\left(\sigma_{2}+\alpha_{2}+\mu\right)}{b \pi}=1>0,
\end{aligned}
$$


Table 1 Indices of sensitivity

\begin{tabular}{lll}
\hline Parameter & Description & Indices \\
\hline$\Re_{0 p}$ & Basic reproduction number of pneumonia & \\
$a$ & Contact rate of pneumonia & $+v e$ \\
$\sigma_{1}$ & Treatment rate of pneumonia & $-v e$ \\
$\alpha_{1}$ & Diseases causing death rate & $-v e$ \\
$\mu$ & Natural causing death rate & $-v e$ \\
$\Re_{0 m}$ & Basic reproduction number of meningitis & \\
$b$ & Contact rate of meningitis & $+v e$ \\
$\sigma_{2}$ & Treatment rate of meningitis & $-v e$ \\
$\alpha_{2}$ & Diseases causing death rate & $-v e$ \\
$\mu$ & Natural causing death rate & $-v e$ \\
\hline
\end{tabular}

$$
\begin{aligned}
& \Lambda_{\alpha_{2}}^{\Re_{0 m}}=\frac{\partial \Re_{0 m}}{\partial \alpha_{2}} \frac{\alpha_{2}}{\Re_{0 m}}=-\frac{\alpha_{2}}{\left(\sigma_{2}+\alpha_{2}+\mu\right)}<0, \\
& \Lambda_{\sigma_{2}}^{\Re_{02}}=\frac{\partial \Re_{0 m}}{\partial \sigma_{2}} \frac{\sigma_{2}}{\Re_{0 m}}=-\frac{\sigma_{2}}{\left(\sigma_{2}+\alpha_{2}+\mu\right)}<0, \\
& \Lambda_{\mu}^{\Re_{0 m}}=\frac{\partial \Re_{0 m}}{\partial \mu} \frac{\mu}{\Re_{0 m}}=-\frac{\left(\sigma_{2}+\alpha_{2}+2 \mu\right)}{\left(\sigma_{2}+\alpha_{2}+\mu\right)}<0 .
\end{aligned}
$$

The above computation of sensitivity analysis is summarized in Table 1.

From Table 1, we understand that the parameters with positive sensitivity indices, particularly $a$ and $b$, have great potential in expanding pneumonia, meningitis, and their coinfection in the community, because they increase their respective reproduction number, which is the average number of a secondary infection. However, the parameters with negative sensitivity index have a great contribution in controlling the expansion of pneumonia and meningitis in the community if their values are increased by keeping other parameters constant.

\section{Numerical simulation}

In this section, some numerical simulation is performed for the full model (pneumoniameningitis co-infection model). We have used Maple 18 for checking the effect of some parameters in the expansion as well as for the control of pneumonia only, meningitis only, and co-infection of pneumonia and meningitis. The parameter values in Table 2 are used for simulation purpose.

\subsection{Effect of recovery rate on pneumonia infectious population}

In this subsection, as we see in Fig. 2, we have experimented on the effect of $\sigma_{1}$ in decreasing the number of pneumonia only infectious population by keeping the contact rate constant $(a=0.9)$. The figure reflects that when the values of $\sigma_{1}$ increase, the number of pneumonia only infectious population is going down. Therefore public policy makers must concentrate on maximizing the values of recovery rate either by treating infected population or by boosting the immunity level of individuals to pneumonia disease.

\subsection{Effect of recovery rate on meningitis infectious population}

In Fig. 3 we see that $\sigma_{2}$ plays a significant role in bringing down the meningitis infection. When the value of $\sigma_{2}$ is increased from 0.1 to 0.9 , the amount of infectious population due to meningitis is decreased, whereas the contact rate is kept constant, which is $b=0.06$. In fighting the meningitis disease, healthy workers or government must give big attention to treating of the infected population in a community. 
Table 2 Parameter values of the model

\begin{tabular}{lll}
\hline Parameter symbol & Value & Source \\
\hline$\delta_{1}$ & $0.003-0.1$ & {$[7]$} \\
$\delta_{2}$ & $0.00904-0.99$ & {$[3]$} \\
$\delta_{3}$ & 0.01 & Assumed \\
$a$ & $0.007-0.6$ & {$[9]$} \\
$b$ & 0.9 & {$[3]$} \\
$\alpha_{1}$ & $0.006-0.5$ & Estimated \\
$\alpha_{2}$ & $0.002-0.2$ & Estimated \\
$\sigma$ & 0.1 & {$[7]$} \\
$g$ & $0.5-1$ & {$[9]$} \\
$e$ & $0.5-1$ & {$[9]$} \\
$\mu$ & 0.01 & Assumed \\
$\sigma_{1}$ & 0.9 & Assumed \\
$\sigma_{2}$ & 0.8 & Assumed \\
\hline
\end{tabular}

Figure 2 Effect of recovery rate on pneumonia infectious population

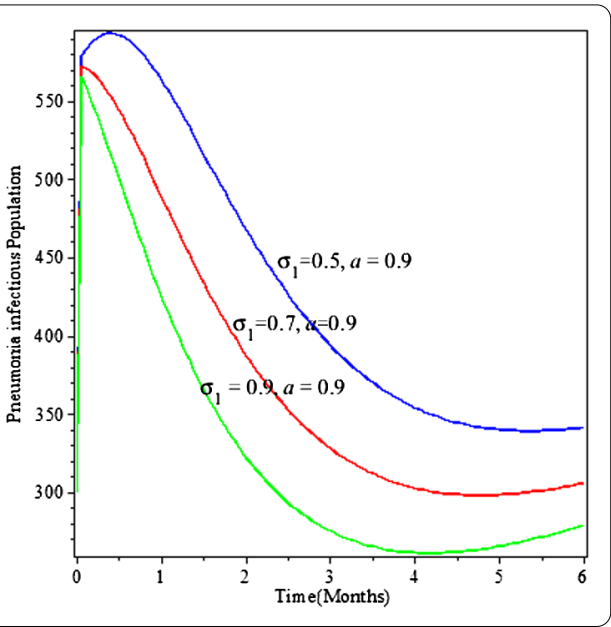

Figure 3 Effect of recovery rate on meningitis infectious population

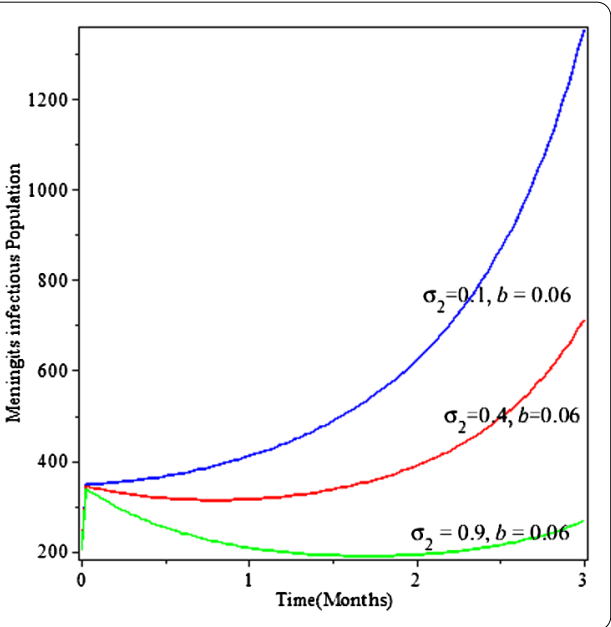

\subsection{Effect of pneumonia contact rate (a) on co-infectious population}

In this section, as we see in Fig. 4, the contact rate of meningitis $(b)$ and the recovery rate of co-infectious population $(\sigma)$ are kept constant. The figure reflects that as the value of contact rate of pneumonia is increased, the co-infectious population increases, which means the expansion of co-infection of pneumonia and meningitis will increase. To con- 
Figure 4 Effect of pneumonia contact rate on co-infectious population

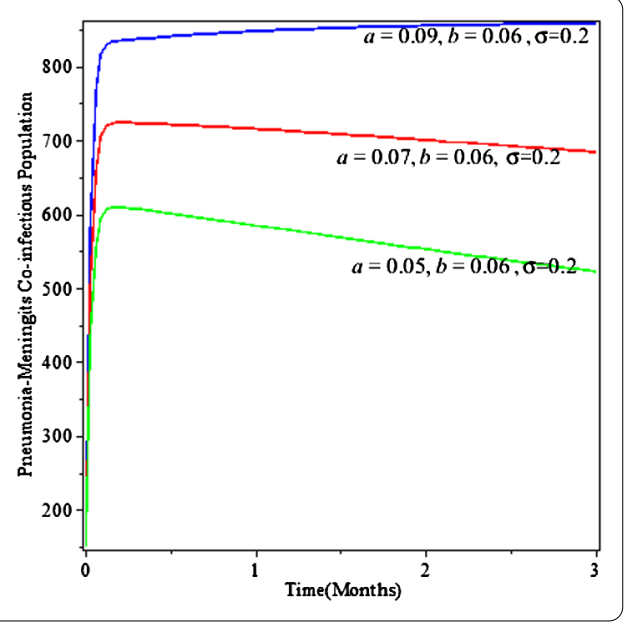

Figure 5 Effect of meningitis contact rate on co-infectious population

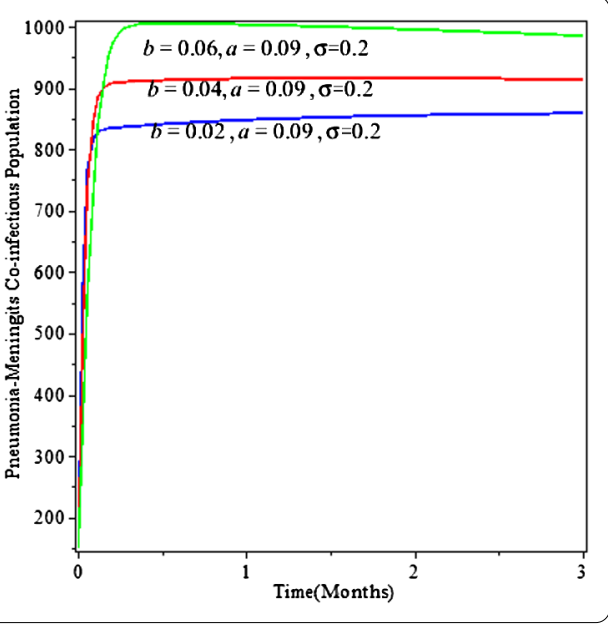

trol co-infection of pneumonia and meningitis, decreasing the contact rate of pneumonia is important. Therefore, stakeholders must concentrate on decreasing the contact rate of pneumonia by quarantine of pneumonia infected or by using an appropriate method of prevention mechanism to bring down the expansion of co-infection in the community.

\subsection{Effect of contact rate of meningitis on co-infectious population}

Similarly, in this section we have investigated the effect of meningitis contact rate $(b)$ in the expansion of pneumonia-meningitis co-infection while keeping the recovery rate of co-infection $(\sigma)$ constant. Figure 5 shows that co-infectious population decreases as the meningitis contact rate is decreasing, by keeping pneumonia contact rate $(a)$ and $\sigma$ constant. This implies that, to bring down co-infection of pneumonia and meningitis, decreasing the contact rate of meningitis is vital.

\subsection{Effect of recovery rate on co-infectious population}

In this subsection, we experimented on the effect of recovery rate of pneumonia and meningitis $(\sigma)$ on the co-infectious population. As we explained in the model description in Sect. 2, due to treatment or other mechanisms, co-infectious population either recover from pneumonia only or from meningitis only or from both diseases with their own 
Figure 6 Effect of recovery rate on co-infectious population

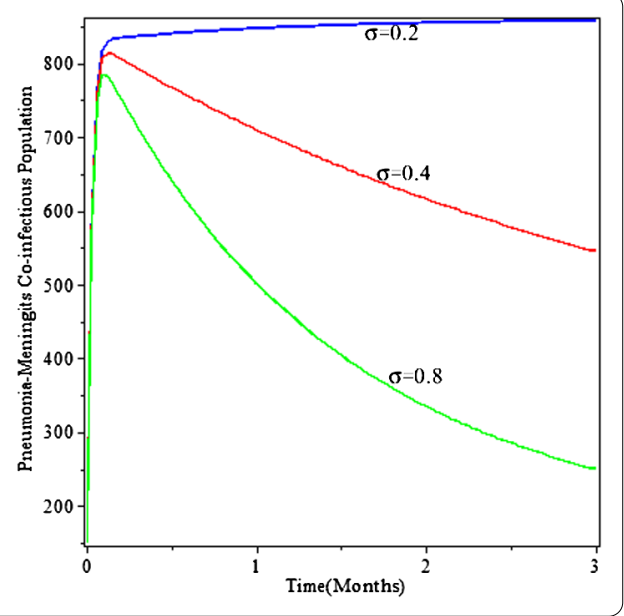

probability and join their respective recovered compartment. Therefore, Fig. 6 shows that increasing the recovery rate of co-infectious population, which is $\sigma$, has a great advantage of eradicating both diseases in the community.

\section{Discussion and conclusion}

In Sect. 2 the proposed model is described in brief. The deterministic model is developed using ordinary differential equation and is sub-divided into seven compartments. In Sect. 3, the model is analyzed qualitatively. To study the qualitative behavior of the model, first we split the full model into two, which are pneumonia only and meningitis only models. The qualitative behaviors, i.e., invariant region of the models, positivist of future solutions of the models, disease-free equilibrium, basic reproduction numbers, endemic equilibria, stability analysis of DFE, and sensitivity analysis of basic reproductions of pneumonia only, meningitis only, and the full model, are analyzed in their respective order. In Sect. 4, numerically, we experimented on the effect of basic parameters in the expansion or control of pneumonia only, meningitis only, and co-infectious diseases. From the result we conclude that increasing the pneumonia recovery rate has a great contribution to bringing down pneumonia infection in the community. Similarly, increasing the meningitis recovery rate also has a contribution of eliminating meningitis diseases. The co-infection recovery rate also has an influence of minimizing co-infectious population if its value is increased. The other result obtained in this section is that decreasing the contact rate of either pneumonia or meningitis has a great influence on controlling coinfection of pneumonia and meningitis in the population.

Acknowledgements

I would like to express my heartfelt appreciation to Haramaya University for financial support and also I am grateful to the anonymous reviewers.

Funding

Not applicable.

Availability of data and materials

The data we used for this research is from respective published articles that are cited. 
Authors' contributions

The main idea of this paper was proposed by GTT. GTT prepared the manuscript initially and performed all the steps of the proof in this research. The author read and approved the final manuscript.

\section{Publisher's Note}

Springer Nature remains neutral with regard to jurisdictional claims in published maps and institutional affiliations.

Received: 12 December 2018 Accepted: 8 April 2019 Published online: 23 April 2019

\section{References}

1. Leach, R.: In: McLuckie, A. (ed.) Respiratory Disease and Its Management, p. 51. Springer, Berlin (2009)

2. WHO: Technical basis for the $\mathrm{WHO}$ recommendations on the management of pneumonia in children at first level health facilities (2008)

3. Fresnadillo Martínez, M.J., García Merino, E., García Sánchez, E., García Sánchez, J.E., Martín del Rey, A., Rodríguez Sanchez, G.: A mathematical model to study the meningococcal meningitis. Proc. Comput. Sci. 18, 2492-2495 (2013)

4. Joseph, E.: Mathematical Analysis of Prevention and Control Strategies of Pneumonia in- Adults and Children. Unpublished MSc Dissertation. University of DareSalaam, Tanzania (2012)

5. Tilahun, G.T., Makinde, O.D., Malonza, D.: Modelling and optimal control of typhoid fever disease with cost-effective strategies. Comput. Math. Methods Med. 2017, Article ID 2324518 (2017). https://doi.org/10.1155/2017/2324518

6. Pessoa, D.: Modelling the dynamics of streptococcus pneumonia transmission in children. Master's thesis, University of De Lisboa (2010)

7. Tilahun, G.T., Makinde, O.D., Malonza, D.: Modelling and optimal control of pneumonia disease with cost-effective strategies. J. Biol. Dyn. 1-27. PMID: 28613986 (2017)

8. Kiddy, J., Asamoah, K., Nyabadza, F., Seidu, B., Chand, M., Dutta, H.: Comput. Math. Methods Med. 2018, Article ID 2657461. https://doi.org/10.1155/2018/2657461

9. Tilahun, G.T., Makinde, O.D., Malonza, D.: Co-dynamics of pneumonia and typhoid fever diseases with cost effective optimal control analysis. Appl. Math. Comput. 316, 438-459 (2018). https://doi.org/10.1016/j.amc.2017.07.063

10. Onyinge, D.O., Ongati, N.O., Odundo, F.: Mathematical Model for co-infection of pneumonia and HIV/AIDS with treatment. Int. J. Sci. Eng. Appl. Sci. 2(1) (2016)

11. Akinyi, O.C., Mugisha J.Y., T., Manyonge, A., Ouma, C.: Modelling the impact of misdiagnosis and treatment on the dynamics of malaria concurrent and co-infection with pneumonia. Appl. Math. Sci. (2013)

12. Lenhart, S.M., Workman, J.T.: Optimal Control Applied to Biological Models. CRC Press, Boca Raton (2007)

13. van den Driessche, P., Watmough, J.: Reproduction numbers and sub-threshold endemic equilibria for compartmental models of disease transmission. Math. Biosci. 180, 29-48 (2002)

14. LaSalle, J.P.: The Stability of Dynamical Systems. SIAM, Philadelphia (1976)

\section{Submit your manuscript to a SpringerOpen ${ }^{\circ}$ journal and benefit from:}

- Convenient online submission

$\checkmark$ Rigorous peer review

- Open access: articles freely available online

- High visibility within the field

- Retaining the copyright to your article

Submit your next manuscript at $>$ springeropen.com 